Alma Mater Studiorum - Università di Bologna DEPARTMENT OF ECONOMICS

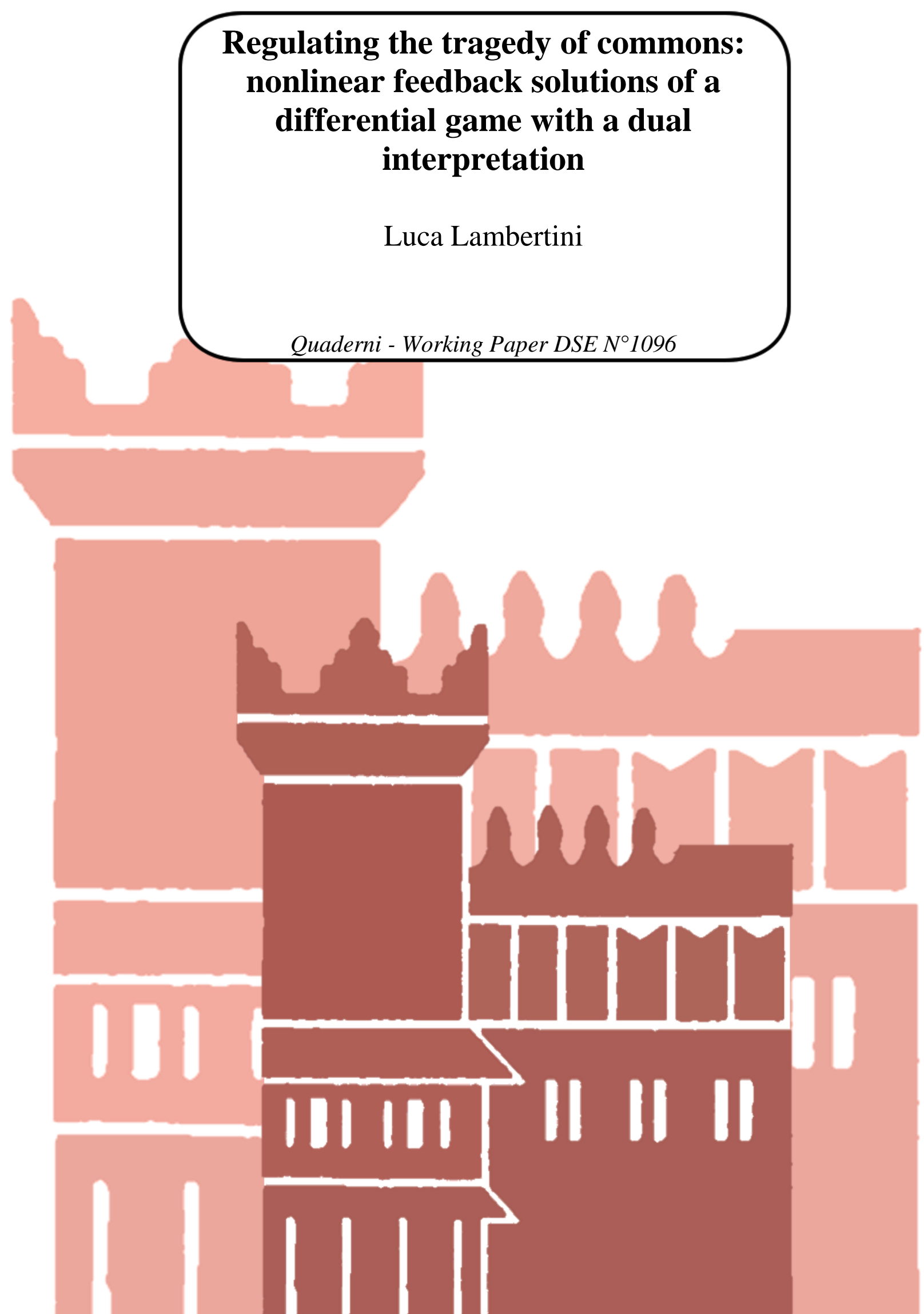




\title{
Regulating the tragedy of commons: nonlinear feedback solutions of a differential game with a dual interpretation $^{1}$
}

\author{
Luca Lambertini \\ Dipartimento di Scienze Economiche \\ Università di Bologna \\ Strada Maggiore 45, 40125 Bologna, Italy \\ luca.lambertini@unibo.it
}

March 9, 2017

\footnotetext{
${ }^{1}$ I would like to thank the seminar audience at the Department of Economics of the University of Salento for helpful comments and discussion. The usual disclaimer applies.
} 


\begin{abstract}
A well established dynamic model describing the impact of oligopolistic interaction on a renewable resource is revisited here to illustrate its dual interpretation as a waste removal differential game. The regulatory implications are illustrated by assuming that the public agency may control market price and possibly also access to the commons. Two different formulations of the managerial or CSR objective are envisaged, based on a combination of profits and either output or the individual share of the waste stock. It is shown that if the representative firm's objective includes the residual waste stock, there exists a unique regulated price driving to zero the steady state stock itself. Hence, the present analysis delivers some useful indications concerning an appropriate definition of the CSR objective firms should adopt.
\end{abstract}

JEL Codes: C73, L13, Q20, Q53

Keywords: waste removal; resource extraction; feedback information; regulation; tragedy of commons 


\section{Introduction}

Free access to the commons is the driver of the original formulation of the tragedy in Gordon (1954) and Hardin (1968). This, in terms of oligopoly games, directly translates into the question as to whether there might exist an optimal industry structure, or, an optimal number of firms in the commons. The analysis of this problem can be traced back to Cornes et al. (1986), Mason et al. (1988) and Mason and Polasky (1997, 2002).

What follows presents in a single model the impact of oligopolistic interaction on a renewable resource and a waste stock via a differential game approach. The idea that originated this paper stems from an elementary analogy between the exploitation of a renewable natural resource and waste removal, provided the dynamics according to which these two magnitudes grow over time can be assumed to be exogenously given and identical. The issue at stake, then, boils down to the following: if the state is a natural resource or species, in line of principle it would be desirable to have the largest possible stock of it left at the steady state, while the opposite holds if the state variable consists of waste. Hence, the policy implications of the ensuing analysis will be opposite in the two cases.

In building up the model, I will pose that firms define their individual objective functions attaching a positive weight to their output levels or harvest rates or, alternatively, to the individual symmetric share of the stock. That is, a firm's objective function is defined as a combination of profits and either the control or the state variable. One way or the other, this approach, in the light of the typical interpretation deriving from an established view in the theory of industrial organization, amounts to saying that firms have separated ownership from control via delegation contracts to managers à la Vickers (1985). However, also in this respect one can spot a dual nature of this additional feature, whereby if the common pool is a stock of waste then 
maximising a combination of profits and output reveals the adoption of a CSR stance by the same firms. ${ }^{1}$

For the sake of simplicity, in the remainder I will quite freely refer to the state variable as a renewable resource or a waste stock, and specify the relevant interpretation of the state when it comes to evaluating the consequences of firms' behaviour, and therefore also the design of an appropriate regulation.

In particular, if the state measures a stock of waste, the ensuing analysis shows that including the state in the maximand is definitely preferable to the alternative based on a combination of profits and individual output (or waste removal). This is because under this specification of the model the regulator avails of a unique regulated price which drives to zero the residual stock associated to any stable equilibria arising under feedback information.

The structure of the paper is the following. The basic setup is laid out in section 2. The first version, where the CSR or managerial objective features the output level, is fully characterised in section 3 , including the unregulated open-loop, linear and nonlinear feedback solutions as well as the regulated feedback game. Section 4 accounts for the linear and nonlinear feedback solutions of the alternative model, for the regulated case only. Plausible extensions and concluding remarks are in section 5 .

\section{The setup}

The setup is an extension of Lambertini and Mantovani (2014) and Benchekroun (2008), where a common property productive asset oligopoly is considered, and encompasses the duopoly model used in Benchekroun (2003) and Fuji-

\footnotetext{
${ }^{1}$ This analogy between strategic delegation and corporate social responsibility has already beeen highlighted in the literature. See Laambertini and Tampieri (2015) and the references therein.
} 
wara (2008). The model illustrates a differential oligopoly game of resource extraction unravelling over continuous time $t \in[0, \infty)$. The market is supplied by $n \geq 1$ firms $^{2}$ producing a homogeneous good, whose inverse demand function is $p=a-Q$ at any time $t$, with $Q=\sum_{i=1}^{n} q_{i}$. Firms share the same technology, characterised by the cost function $C_{i}=c q_{i}^{2}$ in which parameter $c \in(0, a)$ is constant over time. Firms operate without any fixed costs. During production, each firm exploits a renewable natural resource, whose accumulation is governed by the following dynamics:

$$
\dot{S}=F(S)-Q
$$

with

$$
F(S)=\left\{\begin{array}{c}
\delta S \forall S \in\left(0, S_{y}\right] \\
\delta S_{y}\left(\frac{S_{\max }-S}{S_{\max }-S_{y}}\right) \forall S \in\left(S_{y}, S_{\max }\right]
\end{array}\right.
$$

where $S$ is the resource stock, $\delta>0$ is its implicit growth rate when the stock is at most equal to $S_{y}$ and $\delta S_{y}$ is the maximum sustainable yield. Taken together, (1-2) imply that (i) if the resource stock is sufficiently small the population grows at an exponential rate; and (ii) beyond $S_{y}$, the asset grows at a decreasing rate. Moreover, $S_{\max }$ is the carrying capacity of the habitat, beyond which the growth rate of the resource is negative, being limited by available amounts of food and space. In the remainder, we will confine our attention to the case in which $F(S)=\delta S .^{3}$

Firms play noncooperatively and choose their respective outputs simultaneously at every instant. At $t=0$, each firm hires a manager whose contract

\footnotetext{
${ }^{2}$ Under monopoly the delegation to managers would not be operated by stockholders, but CSR could be adopted, so I'm intentionally not ruling out the monopoly case. Another good reason not to do so pops up in section 4 .

${ }^{3}$ As in Benchekroun and Long (2002), Fujiwara (2008) and Tornell and Velasco (1992), among several others.
} 
specifies the instantaneous objective which the manager has to maximise. Delegation contracts are observable. As in Vickers (1985), the delegation contract establishes that the instantaneous objective function of manager $i$ is a linear combination of profits and output: ${ }^{4}$

$$
M_{i}=\pi_{i}+\theta q_{i}
$$

in which $\theta$ determines the relevance of output in the firm's objective.

An alternative approach consists in supposing that the CSR managerial incentive is

$$
M_{i}=\pi_{i}-\theta \cdot \frac{S}{n}
$$

where $\theta$ is a weight attached to the individual symmetric share of the stock. Intuitively, $\theta>0$ seems appropriate if the state is a stock of waste. In both cases, $\theta$ is treated as a constant and is symmetric across the population of firms.

The $i$-th manager maximises the following discounted payoff flow

$$
\Omega_{i}=\int_{0}^{\infty} M_{i} e^{-\rho t} d t
$$

under the constraint posed by the state equation

$$
\dot{S}=\delta S-Q
$$

Parameter $\rho>0$ is the discount rate, common to all managers and constant over time. Obviously, if $\theta=0$, firms behave as pure profit-seeking entrepreneurial units.

The analysis will be carried out under the following assumption:

\footnotetext{
${ }^{4}$ This contract is equivalent to that considered in Fershtman and Judd (1987) and Sklivas (1987), where the maximand is a weighted average of profits and revenues, $M_{i}=$ $\alpha \pi_{i}+(1-\alpha) R_{i}, R_{i}=p q_{i}$. A proof of the equivalence is in Lambertini and Trombetta (2002).
} 
Assumption $1 \delta>\rho[n(n+2 c)+1] /[2(1+c)]$.

This guarantees the positivity of the residual resource stock at the steady state under any feedback rules. That is, in the remainder I will leave the possibility of resource exhaustion due to an excessively large number of firms out of the picture, in order to focus solely on the effects of delegation.

In the remainder of the paper, I will refer to the game relying on (3) as model I, while that using (4) will be model II.

\section{Model I}

Here, delegation (or the adoption of a CSR stance) has the same structure as in Vickers (1985), the instantaneous managerial objective being (3). A few words will suffice to capture the essence of the open-loop solution, which, for several reasons, is of limited interest. In the remainder of this section, I will pose $\sigma \equiv a+\theta$ for the sake of simplicity. If firms don't internalise the consequences of their behaviour at any time and play the individual (static) Cournot-Nash output

$$
q^{C N}=\frac{\sigma}{n+1+2 c}
$$

at all times, then the residual amount of the natural resource in steady state is $S^{C N}=n \sigma /[\delta(n+1+2 c)]=Q^{C N} / \delta$. As the remainder of the analysis is about to show, it is worth noting that the static solution corresponds to the open-loop steady state one, which in this game is unstable (see below). Let the initial condition be $S(0)=S_{0}>0$. The relevance of the size of $S_{0}$ on the final resource stock as well as on the stability of solutions will be discussed in the ensuing analysis. 


\subsection{The linear feedback solution}

The game can be solved under feedback rules conjecturing a linear-quadratic value function with unknown coefficients to be determined solving the resulting system of equations to determine coefficients, or following an alternative but equivalent procedure consisting in solving the relevant first order condition w.r.t. the partial derivative of the value function. For reasons which will become evident below, here I take the latter route. The Hamilton-JacobiBellman (HJB) equation writes as:

$$
\rho V_{i}(S)=\max _{q_{i}}\left[\left(\sigma-Q+c q_{i}\right) q_{i}+V_{i}^{\prime}(S)(\delta S-Q)\right]
$$

where $V_{i}(S)$ is the firm $i$ 's value function; and $V_{i}^{\prime}(S)=\partial V_{i}(S) / \partial S$. The first order condition (FOC) on $q_{i}$ is

$$
\sigma-2(1+c) q_{i}-\sum_{j \neq i} q_{j}-V_{i}^{\prime}(S)=0
$$

In view of the ex ante symmetry across firms, one can impose the symmetry conditions $q_{i}=q(S)$ and $V_{i}(S)=V(S)$ for all $i$ and solve FOC (9) to obtain

$$
V^{\prime}(S)=\sigma-[n+1+2 c] q
$$

Substituting this into (8) yields an identity in $S$. Differentiating both sides with respect to $S$ and rearranging terms, any feedback strategy is implicitly given by the following differential equation:

$$
q^{\prime}(S)=\frac{(\delta-\rho)[\sigma-(n+1+2 c) q(S)]}{\sigma(n-1)+\delta(n+1+2 c) S-2\left[n^{2}+c(2 n-1)\right] q(S)},
$$

which must hold together with terminal condition $\lim _{t \rightarrow \infty} e^{-\rho t} V(s)=0$. Examining expression (11) reveals that

$$
q^{\prime}(S)=0 \Leftrightarrow q_{0}(S)=\frac{\sigma}{n+1+2 c}=\widehat{q}
$$




$$
q^{\prime}(S) \rightarrow \pm \infty \Leftrightarrow q_{\infty}(S)=\frac{\sigma(n-1)+\delta(n+1+2 c) S}{2\left[n^{2}+c(2 n-1)\right]}
$$

Then, assuming that the extraction strategy is a linear function of the stock at any time, I assume $q(S)=\alpha+\beta S$, whereby (11) is satisfied by any pair $(\alpha, \beta)$ solving the following system:

$$
\begin{gathered}
\sigma[\beta(n-1)-\delta+\rho]+\alpha\left[(n+1+2 c)(\delta-\rho)-2\left(n^{2}+c(2 n-1)\right) \beta\right]=0 \\
\beta\left[(n+1+2 c)(2 \delta-\rho)-2\left(n^{2}+c(2 n-1)\right) \beta\right]=0
\end{gathered}
$$

System (14) is solved by the pairs

$$
\begin{gathered}
\alpha_{1}=-\frac{\sigma[2 \delta(1+c)-\rho(n(n+2 c)+1)]}{2 \delta(n+1+2 c)\left[n^{2}+c(2 n-1)\right]} ; \beta_{1}=\frac{(n+1+2 c)(2 \delta-\rho)}{n^{2}\left[n^{2}+c(2 n-1)\right]} \\
\alpha_{2}=\frac{\sigma}{n+1+2 c} ; \beta_{2}=0
\end{gathered}
$$

so that the individual equilibrium output is

$$
\begin{gathered}
q^{L F}(S)=\alpha_{1}+\beta_{1} S \\
q^{O L}=\alpha_{2}=\frac{\sigma}{n+1+2 c}
\end{gathered}
$$

where superscripts $L F$ and $O L$ stand for linear feedback and open-loop, respectively. That is, since the game is a linear state one by construction, one of the linear feedback strategies generated by the HJB equation degenerate in the open-loop one, coinciding with the static Cournot-Nash solution. ${ }^{5}$ The expression on the r.h.s. of $(17)$ belongs to $[0, \sigma /(n+1+2 c)]$ for all

$$
S \in\left[\frac{\sigma[n(n+2 c)+1]}{\delta(n+1+2 c)^{2}}, \frac{\sigma[2(1+c) \delta-\rho(n(n+2 c)+1)]}{(n+1+2 c)^{2}(2 \delta-\rho) \delta}\right]
$$

If $q=q^{L F}(S)$, the steady state level of the natural resource stock is

$$
S^{L F}=\frac{n \sigma[2(1+c) \delta-\rho(n(n+2 c)+1)]}{\delta(n+1+2 c)[2 c(\delta-n \rho)+n(2 \delta-\rho(n+1))]}>0
$$

\footnotetext{
${ }^{5}$ For moere on classes of differential games in which the open-loop solution is subgame perfect (or strongly time consistent), see Dockner et al. (1985), Fershtman (1987), Mehlmann (1988), Dockner et al. (2000) and Cellini et al. (2005).
} 
for all values of $\delta$ satisfying Assumption 1. It is evident that $\partial S^{L F} / \partial \theta>0$ since $\partial S^{L F} / \partial \sigma>0$. That is,

Lemma 1 At the linear feedback equilibrium, any increase in the extent of delegation increases the residual stock of resources in steady state.

If instead $q=q^{O L}$, the steady state level of the natural resource stock associated with open-loop strategies is

$$
S^{O L}=\frac{n \sigma}{\delta(n+1+2 c)}>0
$$

everywhere.

Solutions $q^{O L}$ and $q^{L F}(S)$, together with the locus $\dot{S}=0$, are represented in the space $(q, S)$ in Figure 1, where arrows illustrate the dynamics of variables and the stability of $q^{L F}(S)$, as opposed to the instability of the open-loop solution $q^{O L}$. If firms adopt this strategy, the resource stock is bound to shrink to zero for all $S_{0}<S^{O L}$. Otherwise, for all $S_{0}>S^{O L}$, the stock will grow beyond $S_{y}$, not represented along the horizontal axis of Figure 1. Hence, under open-loop rules, the ultimate destiny of the natural resource depends on initial conditions. It is also worth stressing that $\partial S^{O L} / \partial \theta>0$, which implies that the interval of initial conditions leading to resource extinction under open-loop (or quasi-static) strategies expands in the extent of managerial delegation. 
Figure 1 Open-loop and linear feedback solutions in the $(S, q)$ space

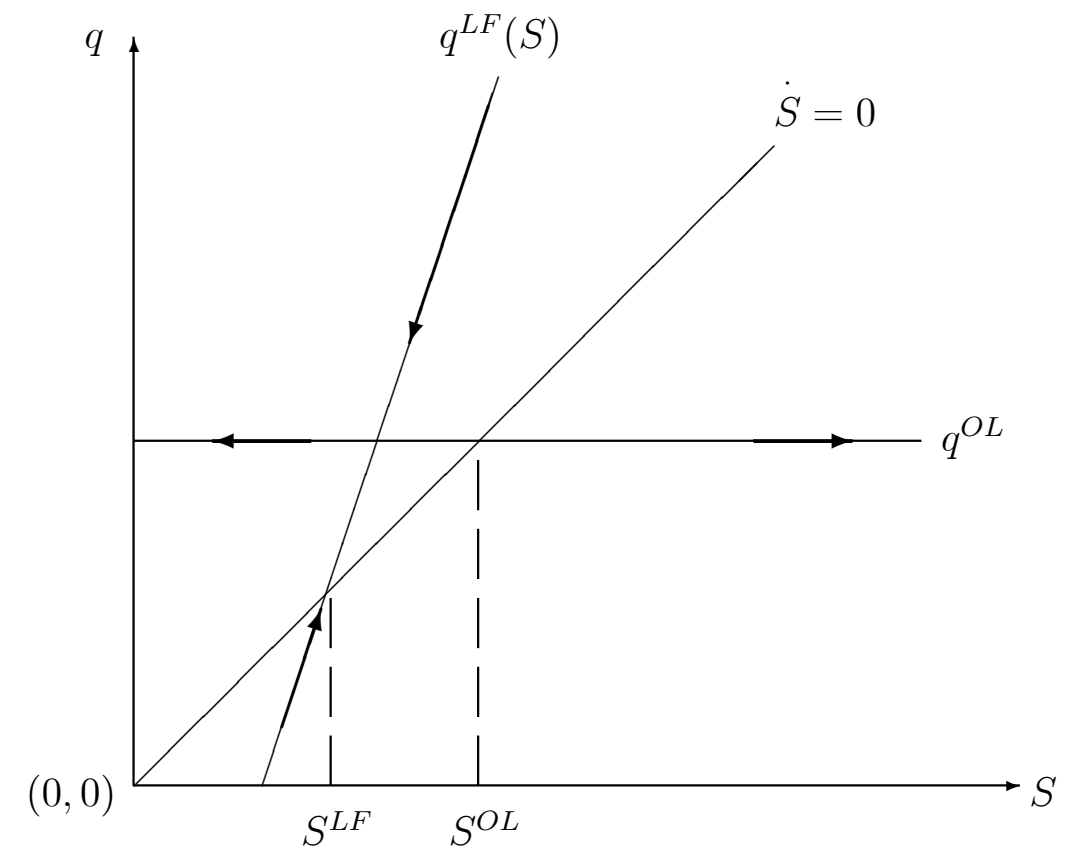

\subsection{Nonlinear feedback equilibria}

The present game produces infinitely many nonlinear feedback solutions whose continuum can be fully characterised using the same procedure as in Lambertini (2016a) and Lambertini and Mantovani (2016), which in turn relies on Rowat's (2007). ${ }^{6}$ Without replicating the entire analysis of the nonlinear case, here it suffices to characterise the degenerate nonlinear solution identified by the tangency between the highest isocline of the representative

\footnotetext{
${ }^{6}$ Nonlinear feedback solutions have been investigated in oligopoly theory, environmental and resource economics and other fields. See Tsutsui and Mino (1990), Shimomura (1991), Dockner and Sorger (1996), Itaya and Shimomura (2001), Rubio and Casino (2002) and Colombo and Labrecciosa (2015), inter alia.
} 
firm and the locus $\dot{S}=0$ in the state-control space.

To do so, one has to go back to (11) and note that the slope of the steady state locus $\dot{S}=0$ is

$$
\left.\frac{\partial q(S)}{\partial S}\right|_{\dot{S}=0}=\frac{\delta}{n}
$$

which must coincide with $q^{\prime}(S)$ when $q(S)=\delta S / n$, in such a way that (11) becomes:

$$
\frac{\delta}{n}=\frac{(\delta-\rho)[n \sigma-\delta(n+1+2 c) S]}{(n-1)[n \sigma-\delta(n+2 c) S]}
$$

whose unique solution w.r.t. the state variable is

$$
S^{N L T}=\frac{n \sigma(\delta-n \rho)}{\delta[2 c(\delta-n \rho)+n(2 \delta-\rho(n+1))]}
$$

which is positive in the parameter range wherein $S^{L F}>0$. The associated individual output is $q^{N L T}=\delta S^{N L T} / n$. Superscript $N L T$ mnemonics for nonlinear tangency solution.

Figure 2 describes the evolution of state and control variables over time, enabling one to single out the properties of any nonlinear feedback solutions, including the very specific one generated by the tangency point with the locus $\dot{S}=0$ (point $T$ in the figure). Figure 2 (which is nothing but a more detailed version of Figure 1) also portrays the loci $q^{\prime}(S)=0$ (along which $\left.q_{0}(S)=q^{F 1}(S)=q^{O L}\right)$ and $q^{\prime}(S) \rightarrow \infty$. 
Figure 2 Linear and nonlinear feedback solutions in the $(S, q)$ space

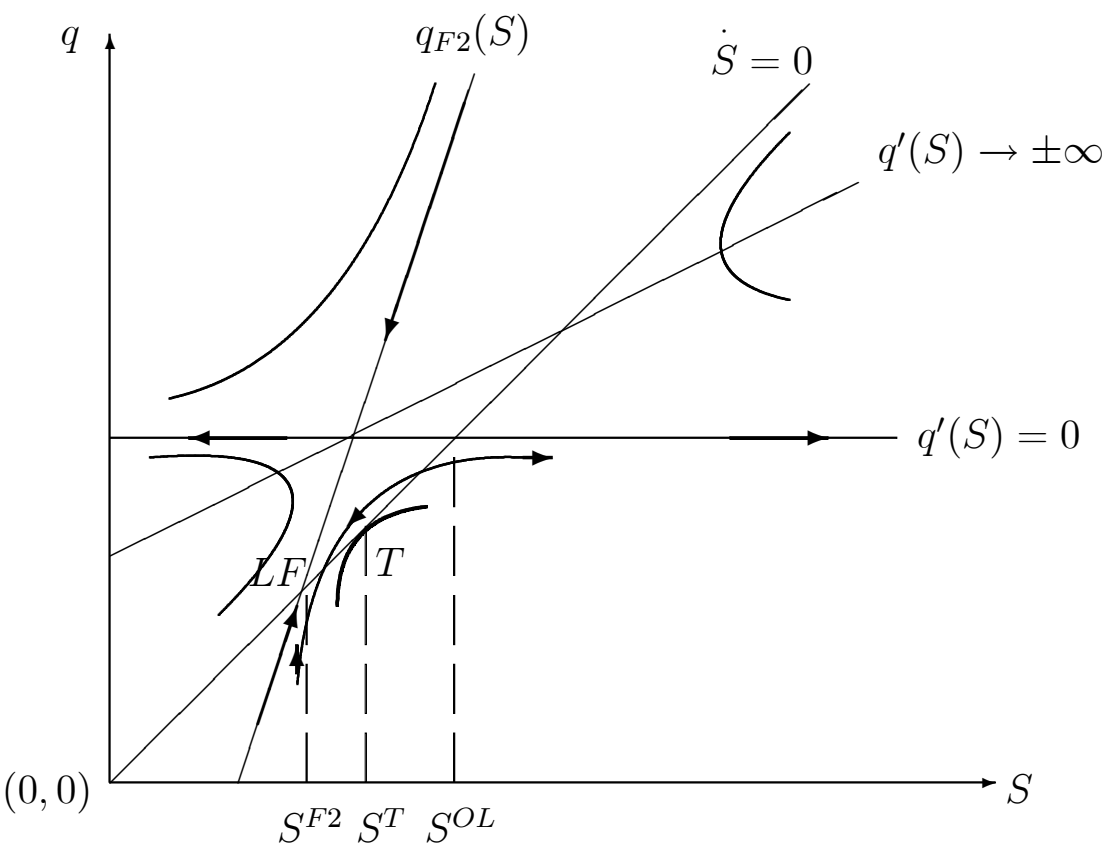

The arrows along the curve tangent to the locus $\dot{S}=0$ in point $T$ shows that the tangency solution is indeed unstable. However, there exist infinitely many solutions identified by the intersections along the segment delimited by points $T$ and $L F$. This set of stable nonlinear solutions, which can be labelled as $S N L S$, is sensitive to the extent of delegation $\theta$, which affects the loci $\dot{S}=0$ and $q^{F 2}(S)$, and therefore also the position of the tangency point $T$. The set $S N L S$ has the size of such a segment:

$$
S N L S=\sqrt{\left(S^{N L T}-S^{L F}\right)^{2}+\left(q^{N L T}-q^{L F}\right)^{2}}
$$

Using the corresponding expressions for the steady state values of state and control, one obtains $S N L S=\sigma \sqrt{\Phi(n, \delta, \rho)}$, with $\Phi(\cdot)>0$. Consequently,

$$
\frac{\partial S N L S}{\partial \theta}=\sqrt{\Phi(n, \delta, \rho)}>0
$$


by the definition of $\sigma$. This boils down to the following:

Proposition 2 The separation between ownership and control via delegation contracts based on output expansion enlarges the set of stable nonlinear feedback solutions.

In particular, since $\partial S^{N L T} / \partial \theta>0$, the above proposition is accompanied by a relevant corollary:

Corollary 3 The adoption of managerial incentives based on output expansion increases the upper bound of the SNLS set.

This result can be rephrased to say that these particular type of managerial incentives allows for a larger stock of the resource surviving in correspondence of a nonlinear feedback solution, and prompts for the analysis of the so-called voracity effect (Lane and Tornell, 1996; Tornell and Lane, 1999), which can be briefly summarised as follows. In line of principle, one would expect that the higher the resource growth rate is, the higher should be the volume of that resource in steady state. However, this may not hold true as firms respond to any increase in the growth rate by hastening resource extraction, whereby one observes that $\partial S / \partial \delta<0$ in steady state, at least for sufficiently high levels of $\delta$. The arising of such voracity effect has been highlighted, with pure profit-seeking units, in Benchekroun (2008) and Lambertini and Mantovani (2014). As in Lambertini and Mantovani (2014, p. 121), also here it can be easily shown that under linear and nonlinear feedback information the voracity effect operates.

Take the weighted average of $S^{L F}$ and $S^{N L T}$ :

$$
\bar{S}=\phi S^{L F}+(1-\phi) S^{N L T}
$$

with $\phi \in[0,1]$. There emerges that $\partial \bar{S} / \partial \delta<0$ for sufficiently high levels of the growth rate $\delta$, for any $\phi \in[0,1]$, thereby including the extremes of 
the relevant interval of resource stock volumes in steady state. However, this property, combined with Lemma 1, Proposition 2 and Corollary 3, entails

Proposition 4 Managerial incentives allowing for output expansion soften the voracity effect over the entire interval of nonlinear feedback solutions SNS.

It would be tempting to interpret this conclusion as implying a beneficial effect of managerialization on resource preservation (or, an undesirable effect upon waste removal, in which case voracity is most welcome for intuitive reasons). However, this would be hazardous as the same issue should indeed be reassessed in presence of alternative incentive schemes, based for instance on market shares (Jansen et al., 2007; Ritz, 2008) or comparative performance evaluation (Salas Fumas, 1992; Miller and Pazgal, 2001). Yet, the possibility that delegating control to agents interested in expanding production might ultimately mitigate the pressure on the resource is a striking and unexpected feature of the present model. This fact finds its explanation in the multiplicative effect of this form of delegation on equilibrium outputs and the resource stock, as the delegation parameter $\theta$ appears in market size $\sigma$ and makes it larger as seen from the managers' standpoint. Since $\sigma$ is a at the same time a measure of profitability or demand level, this type of delegation (i) increases the maximum mark-up from $a-c$ to $a-c+\theta$ or equivalently (ii) shifts the demand upwards by $\theta$. Consequently, the managerial inclination to expanding output is routed in the direction of affecting the mark-up level and this mechanism operates as a partial remedy to voracity in the range where the latter takes place. Therefore, albeit with some caution, this design of delegation contracts - admittedly, far from being general - is of public interest because it couples the usual elements connected with consumer surplus and profits with additional motives (perhaps more far-reaching) dealing with the impact of the separation between ownership and control on resource (and 
species) preservation.

Moreover, there remains the open question as to how a public agency could regulate access to the commons, in presence of a single stable linear feedback equilibrium and infinitely many stable nonlinear feedback equilibria. A plausible solution is proposed in the next section.

\subsection{The regulated case}

The model remains the same as for the resource dynamics (6) and firms' technology. Instead, here the price $p$ is exogenously given, being a policy instrument in the hands of a public authority in charge of regulating access to the common resource pool.

Accordingly, firm $i$ 's instantaneous maximand writes

$$
M_{i}(t)=\left[p-c q_{i}(t)+\theta\right] q_{i}(t) .
$$

The problem is formally defined as above, as firm $i$ 's HJB equation is

$$
\rho V_{i}(S)=\max _{q_{i}}\left\{M_{i}+\frac{\partial V_{i}(S)}{\partial S} \cdot(\delta S-Q)\right\}
$$

Solving the game on the basis of the same procedure (or equivalently using the method of the undetermined parameters), one obtains the following pair of strategies:

$$
q_{p}^{O L}=\frac{\sigma_{p}}{2 c} ; q_{p}^{L F}=\frac{2 c \delta(2 \delta-\rho) S-(\delta-n \rho) \sigma_{p}}{2 c \delta(2 n-1)}
$$

where (i) $\sigma_{p} \equiv p+\theta$; (ii) superscripts have the same meaning as above; and (iii) subscript $p$ indicates that the price of the final good is being regulated. While $q_{p}^{O L}>0$ over the entire parameter space, $q_{p}^{L F}>0$ for all ${ }^{7}$

$$
S>\frac{(\delta-n \rho) \sigma_{p}}{2 c \delta(2 \delta-\rho)}>0
$$

\footnotetext{
${ }^{7}$ La demonstration that indeed

$$
\frac{(\delta-n \rho) \sigma}{2 c \delta(2 \delta-\rho)}>0
$$
}


The interesting implication of the price regulation is that, irrespective of the information structure underpinning firms' strategies, the residual steady state resource stock is exactly the same:

$$
S_{p}=\frac{n q_{p}^{L F, O L}}{\delta}=\frac{n \sigma_{p}}{2 c \delta}
$$

which amounts to saying the following:

Proposition 5 Regulating price eliminates the multiplicity of stable feedback equilibria, with the single linear feedback one surviving.

Moreover, (32) has two relevant implications that should equally attract the attention of the authority:

- $S_{p}$ monotonically increases in $n$ : hence, the minimum residual stock obtains in correspondence of $n=1$. Recalling the dual interpretation of the nature of $S$, this fact has completely opposite implications concerning the socially efficient access to the commons.

- $S_{p}$ monotonically increases in $\sigma$ and therefore also in the extent of delegation, $\theta$ : this reveals that including the individual instantaneous harvest rates in the delegation contracts (or, adopting a CSR stance) might or mighty not mean good news from the regulator's standpoint, again in view of the dual interpretation of the model as for the nature of the state variable.

Be that as it may, the picture looks as in Figure 3, where again the arrows indicate the dynamics of the state $S$ and illustrate that $q_{p}^{O L}$ is unstable while $q_{p}^{L F}$ is stable. Therefore, although they seem to yield the same steady state, derives from the solution of the model in which firms are pure-profit-seeking agents (i.e., $\theta=0$ ) and price is endogenously determined via the linear demand function instant by instant. 
open-loop and feedback information structures are not equivalent at all. In particular, the outcome engendered by $q_{p}^{O L}$ can either drop to $S=0$ or exceed $S_{y}$, depending on the initial stock, ${ }^{8}$ while the volume of the long-run equilibrium state variable generated by $q_{p}^{L F}$ is surely $S_{p}=n \sigma_{p} /(2 c \delta)$.

Figura 3 The regulated case

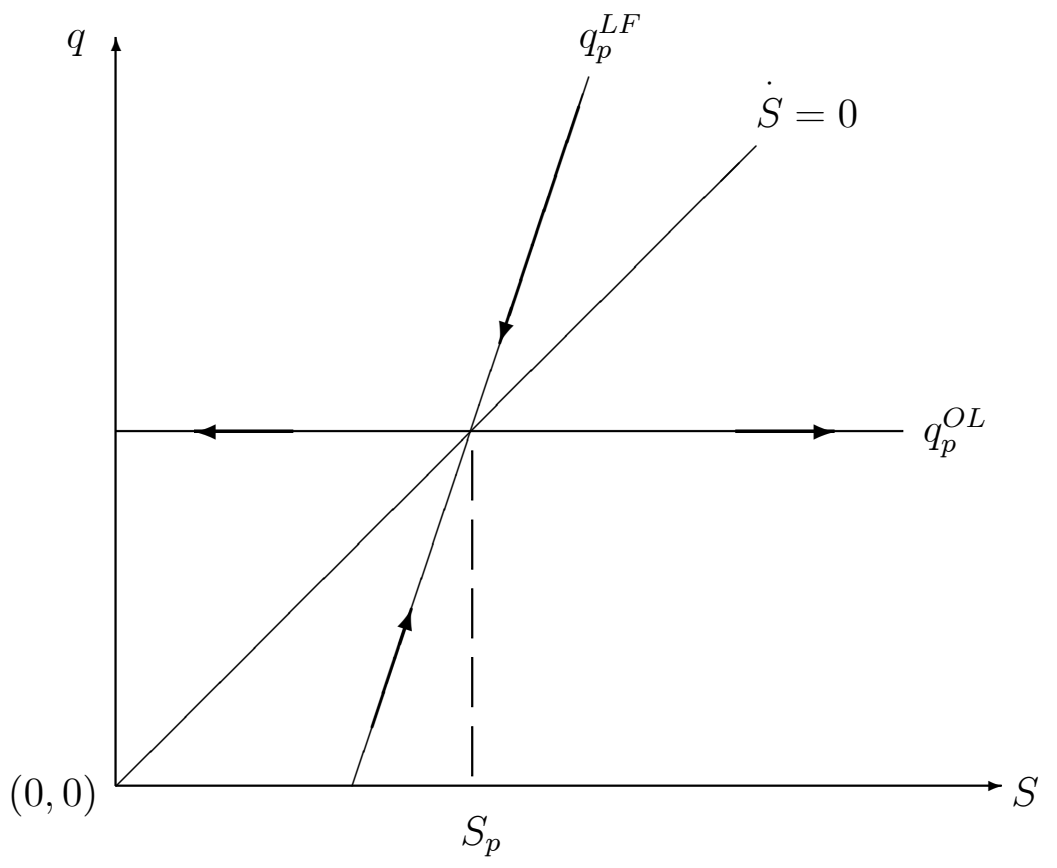

To close the discussion carried out in this section, let's focus our attention onto the case in which $S$ is a stock of waste. If so, then monopoly is the

\footnotetext{
${ }^{8} \mathrm{~A}$ peculiar and somewhat paradoxical feature of the case of waste removal is that if the initial stock is sufficiently low, firms might involuntarily drive to zero the residual stock under myopic open-loop rules. Of course it is also true that if the inital stock is large then the adoption of open-loop strategies might cause the waste stock to shoot up to plus infinity.
} 
socially efficient structure, with

$$
\left.S^{N L T}\right|_{n=1}=\left.S^{L F}\right|_{n=1}
$$

for obvious reasons, and

$$
\left.S^{L F}\right|_{n=1}-\left.S_{p}\right|_{n=1}=\frac{a c-\theta-p(1+c)}{2 \delta c(1+c)}>0
$$

for all

$$
p<\min \left\{0, \frac{a c-\theta}{1+c}\right\}
$$

which entails that waste removal in monopoly should be subsidised if $\theta>a c$.

\section{Model II}

Here, the contract based on (4) says that the firm attaches a negative weight to the residual individual share of waste at the symmetric equilibrium. I will focus on the regulated case only, as here - unlike what we have seen in model I - the continuum of stable feedback equilibria arising with an unregulated price survives the regime change. Hence, in what follows it is assumed that $p$ is an instrument in the regulator's hands. Additionally, for reasons which will become apparent below, I will confine myself to the case in which the state variable is a stock of waste (or, equivalently, the control of all firms has been delegated to CSR managers).

Solving this game under feedback information yields infinitely many subgame perfect strategies. This seemingly undesirable feature is driven by the fact that the HJB equation is solved by the following two linear feedback strategies:

$$
q_{I I}^{a}=\frac{n p(\delta-\rho)-\theta}{2 c n(\delta-\rho)} ; q_{I I}^{b}=\frac{n\left[n p \rho+4 c \delta^{2} S+2 \theta-\delta(p+2 c \rho S)\right]-\theta}{2 c n \delta(2 n-1)}
$$


which produce two different values of the residual waste stock in steady state:

$$
S_{I I}^{a}=\frac{n p(\delta-\rho)-\theta}{2 c \delta(\delta-\rho)} ; S_{I I}^{b}=\frac{n p(\delta-n \rho)-\theta(2 n-1)}{2 c \delta(\delta-n \rho)}
$$

The resulting graph replicates the picture appearing in Figure 1, with analogous properties. In particular, also here the first (open-loop) solution is unstable, while the second is stable. Of course, there are infinitely many nonlinear solutions, a subset of which is stable. This is portrayed in Figure 4, which, except for labels, is the same as in Figure 2.

Figure 4 Non linear solutions in the alternative model

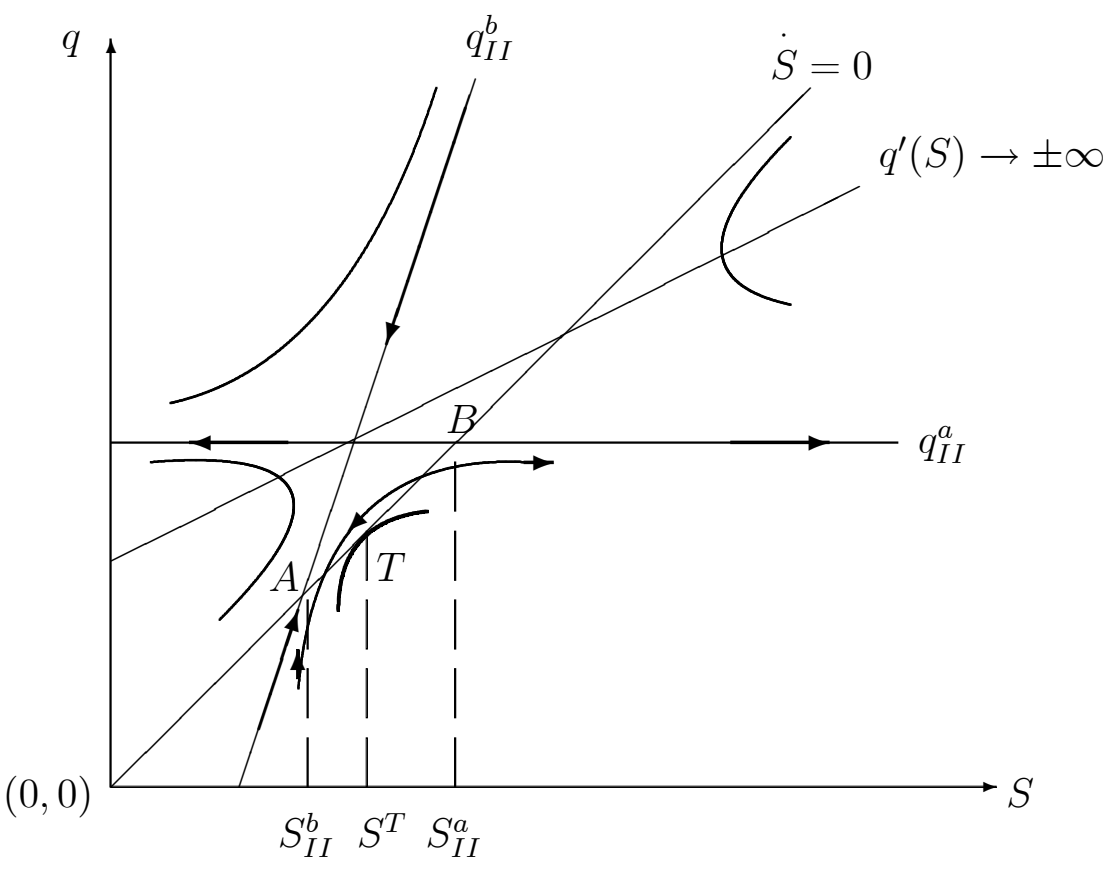

Hence, it is evident that regulation does not deliver uniqueness if the delegation contract (or the CSR stance) chosen by firms relies on the residual stock of waste. Yet, at a closer look, this scenario is not as discouraging as it might look at first glance. To grasp the intuition why it is not so, observe 
that

$$
S_{I I}^{a}-S_{I I}^{b}=\frac{(n-1)(2 \delta-\rho) \theta}{2 c \delta(\delta-\rho)(\delta-n \rho)}>0
$$

for all $\theta>0$. This simple result can be formulated as follows:

Lemma 6 If firms adopt a CSR stance based on a negative weight attached to the individual share of residual waste stock, the stable feedback solution yields a lower residual stock than the unstable (open-loop) one.

This fact has several relevant implications: (i) any stable nonlinear solution is more desirable than the open-loop one; (ii) unlike what happens in models dealing with natural resource exploitation, here the voracity effect (Tornell and Velasco, 1992; Lane and Tornell, 1996; Tornell and Lane, 1999) combined with feedback information is indeed desirable; and, more importantly, both $S^{T}$ and $S_{I I}^{b}$ are monotonically decreasing in $\theta$. That is,

Proposition 7 Intensifying the CSR component in the firm's objective function brings about a decrease in the residual waste shock in any stable steady state reached through feedback strategies.

It is worth noting that this is the opposite of what happens in the previous model, where (32) measures the residual stock. Last but not least, one may verify that for any $\theta>0$ there exists a unique level of the regulated price at which $S_{I I}^{b}=0$ :

$$
p\left(S_{I I}^{b}=0\right)=\frac{\theta(2 n-1)}{n(\delta-n \rho)}>0
$$

with

$$
\frac{\partial p\left(S_{I I}^{b}=0\right)}{\partial n}=\frac{[\delta+2 n(n-1) \rho] \theta}{n^{2}(\delta-n \rho)^{2}}>0 .
$$

The same applies for any equilibrium generated by nonlinear feedback strategies, whose residual stock is $\widehat{S}=\alpha S_{I I}^{b}+(1-\alpha) S^{T}, \alpha \in(0,1)$, as both $S_{I I}^{b}$ and $S^{T}$ are linear in $p$. Moreover, () implies that, in monopoly, $S_{I I}^{a}=S_{I I}^{b}$ and 
therefore the infinitely many nonlinear equilibria vanish. These last findings can be summarised as follows:

Corollary 8 To any stable feedback solution is associated a single price driving to zero the residual waste stock at equilibrium. This price takes its minimum value in monopoly, where the stable linear solution is the only one being relevant as the continuum of nonlinear equilibria disappears.

This suggests that the regulator may indeed rely on a single firm, granting it the lowest price identified by $\left.p\left(S_{I I}^{b}=0\right)\right|_{n=1}$. This simultaneously solves the problem associated with the multiplicity of equilibria and ensures full removal at the lowest cost for society. Of course we are not compelled to take this conclusion literally, in the sense that the correct implementation of the price $p\left(S_{I I}^{b}=0\right)$ requires knowing the exact values of the set of parameters $\{n, \delta, \rho, \theta\}$. However, the sign of partial derivatives (39-40) represents a reliable qualitative indication for the regulator.

\section{Concluding remarks}

In a nutshell, the foregoing analysis has shown that the acquired model describing the dynamic exploitation of a common pool renewable resource could be reinterpreted as a game of waste removal, changing a few labels. Of course, this involves a non trivial change of perspective, in particular when it comes to the need of regulating an oligopoly game generating a continuum of feedback equilibria. Firms are either managerial or CSR entities - depending on the interpretation being chosen - and their objective been defined in two different ways. The first formulation stipulates that the relevant objective contains profits and output (or, the instantaneous individual volume of waste removal). In this case, the adoption of feedback information generates a continuum of stable subgame perfect equilibria. The choice of regulating price 
sweeps away the continuum of equilibria engendered by nonlinear strategies, leaving the regulator with a single stable linear feedback equilibrium whose performance depends on the price level and the number of firms being granted access to the commons. Hence, there appears that, combining appropriately price and entry regulation, the public authority can indeed outperform the most favourable unregulated feedback equilibrium in terms of the residual resource stock at the steady state.

The second formulation assumes that managerial or CSR incentives are based on a combination of profits and the firm's individual share of the residual stock of the state variable. If the latter measures the volume of waste, the model shows that, in correspondence of any stable feedback equilibrium, there exists a price at which the residual stock is indeed nil. Moreover, such a price decreases monotonically in the number of firms. Hence, the regulator may restrict access to a single firm and adopt the lowest of all such prices, thereby attaining the desired goal at the lowest possible tariff.

Needless to say, the foregoing material does not exhaust the analysis of this topic. In addition to the obvious extensions accounting for the aforementioned alternative delegation contracts based upon market shares (Jansen et al., 2007; Ritz, 2008) or comparative performance evaluation (Salas-Fumas, 1992; Miller and Pazgal, 2001), a plausible and promising one is that in which either control variables or the stock implies polluting emissions. The first possibility is plausible if the state refers to a natural resource, and production based on harvest is polluting the environment; the second is intuitively related to a scenario in which the state variable is a stock of waste. This extension would enrich the currently scant literature modelling the simultaneous presence of resource extraction (or stock removal) and environmental damage or global warming (cf. Lambertini and Leitmann, 2013; and Lambertini, 2016b). 


\section{References}

[1] Benchekroun, H. (2003). Unilateral production restrictions in a dynamic duopoly. Journal of Economic Theory 111, 214-39.

[2] Benchekroun, H. (2008). Comparative dynamics in a productive asset oligopoly. Journal of Economic Theory 138, 237-61.

[3] Benchekroun, H. and N.V. Long (2002). Transboundary fishery: a differential game model. Economica 69, 207-21.

[4] Cellini, R., L. Lambertini and G. Leitmann (2005). Degenerate feedback and time consistency in differential games, in E.P. Hofer and E. Reithmeier (eds), Modeling and Control of Autonomous Decision Support Based Systems. Proceedings of the 13th International Workshop on Dynamics and Control, Aachen, Shaker Verlag, 185-92.

[5] Colombo, L. and P. Labrecciosa (2015). On the Markovian efficiency of Bertrand and Cournot equilibria. Journal of Economic Theory 155, $322-58$.

[6] Cornes, R., C.F. Mason and T. Sandler (1986). The commons and the optimal number of firms. Quarterly Journal of Economics, 101, 641-46.

[7] Dockner, E., G. Feichtinger and S. Jørgensen (1985). Tractable classes of nonzero-sum open loop Nash differential games. Journal of Optimization Theory and Applications, 45, 179-97.

[8] Dockner, E.J., S. Jørgensen, N.V. Long and G. Sorger (2000). Differential Games in Economics and Management Science, Cambridge, Cambridge University Press. 
[9] Dockner, E.J. and G. Sorger (1996). Existence and properties of equilibria for a dynamic game on productive assets. Journal of Economic Theory 171, 201-27.

[10] Fershtman, C. (1987). Identification of classes of differential games for which the open-loop is a degenerate feedback Nash equilibrium", Journal of Optimization Theory and Applications, 55, 217-31.

[11] Fershtman, C. and K. Judd (1987). Equilibrium incentives in oligopoly. American Economic Review, 77, 927-40.

[12] Fujiwara, K. (2008). Duopoly can be more anti-competitive than monopoly. Economics Letters 101, 217-19.

[13] Gordon, H.S. (1954). The economic theory of a common-property resource: the fishery. Journal of Political Economy, 62, 124-42.

[14] Hardin, G. (1968). The tragedy of the commons. Science 162, 1243-48.

[15] Itaya, J. and K. Shimomura (2001). A dynamic conjectural variations model in the private provision of public goods: a differential game approach. Journal of Public Economics 81, 153-72.

[16] Jansen, T., A. van Lier and A. van Witteloostuijn (2007). A note on strategic delegation: the market share case. International Journal of Industrial Organization, 25, 531-39.

[17] Lambertini, L. (2016a). Managerial delegation in a dynamic renewable resource oligopoly. In H. Dawid, K. Doerner, G. Feichtinger, P. Kort and A. Seidl (eds), Dynamic Perspectives on Managerial Decision Making: Essays in Honor of Richard F. Hartl, Heidelberg, Springer.

[18] Lambertini, L. (2016b). On the interplay between resource extraction and polluting emissions in oligopoly. In A. Espinola-Arredondo and F. 
Munoz-Garcia (eds), Game Theory Applications to Environmental Economics, Singapore and New York, World Scientific.

[19] Lambertini, L. and G. Leitmann (2013). Market power, resource extraction and pollution: some paradoxes and a unified view. In J. Crespo Cuaresma, T. Palokangas and A. Tarasjev (eds), Green Growth and Sustainable Development, Heidelberg, Springer.

[20] Lambertini, L. and A. Mantovani (2014). Feedback equilibria in a dynamic renewable resource oligopoly: pre-emption, voracity and exhaustion. Journal of Economic Dynamics and Control, 47, 115-22.

[21] Lambertini, L. and A. Mantovani (2016). On the (in)stability of nonlinear feedback solutions in a dynamic duopoly with renewable resource exploitation. Economics Letters, forthcoming.

[22] Lambertini, L. and A. Tampieri (2015). Incentive, performance and desirability of socially responsible firms in a Cournot oligopoly. Economic Modelling, 50, 40-48.

[23] Lambertini, L. and M. Trombetta (2002). Delegation and firms' ability to collude. Journal of Economic Behavior and Organization, 47, 359-73.

[24] Lane, P.R. and A. Tornell (1996). Power, growth, and the voracity effect. Journal of Economic Growth 1, 213-41.

[25] Mason, C.F. and S. Polasky (1997). The optimal number of firms in the commons: a dynamic approach. Canadian Journal of Economics, 30, 1143-60.

[26] Mason, C. and S. Polasky (2002). Strategic preemption in a common property resource: a continuous time approach. Environmental and Resource Economics, 23, 255-78. 
[27] Mason, C., T. Sandler and R. Cornes (1988). Expectations, the commons, and optimal group size. Journal of Environmental Economics and Management, 15, 99-110.

[28] Mehlmann, A. (1988). Applied Differential Games, New York, Plenum Press.

[29] Miller, N.H. and A.I. Pazgal (2001). The equivalence of price and quantity competition with delegation. RAND Journal of Economics, 32, 284301.

[30] Ritz, R. (2008). Strategic incentives for market share. International Journal of Industrial Organization, 26, 586-97.

[31] Rowat, C. (2007). Non-linear strategies in a linear quadratic differential game. Journal of Economic Dynamics and Control 31, 3179-202.

[32] Rubio, S.J., and B. Casino (2002). A note on cooperative versus noncooperative strategies in international pollution control. Resource and Energy Economics 24, 251-61.

[33] Salas Fumas, V. (1992). Relative performance evaluation of management: the effects on industrial competition and risk sharing. International Journal of Industrial Organization, 10, 473-89.

[34] Shimomura, K. (1991). The feedback equilibria of a differential game of capitalism. Journal of Economic Dynamics and Control 15, 317-38.

[35] Sklivas, S. (1987). The strategic choice of management incentives. RAND Journal of Economics, 18, 452-58.

[36] Tornell, A. and P.R. Lane (1999). The voracity effect. American Economic Review 89, 22-46. 
[37] Tornell, A. and A. Velasco (1992). The tragedy of the commons and economic growth: why does capital flow from poor to rich countries? Journal of Political Economy, 100, 1208-31.

[38] Tsutsui, S. and K. Mino (1990). Nonlinear strategies in dynamic duopolistic competition with sticky prices. Journal of Economic Theory $\mathbf{5 2}, \mathbf{1 3 6 - 6 1 .}$

[39] Vickers, J. (1985). Delegation and the theory of the firm. Economic Journal (Conference Supplement), 95, 138-47. 


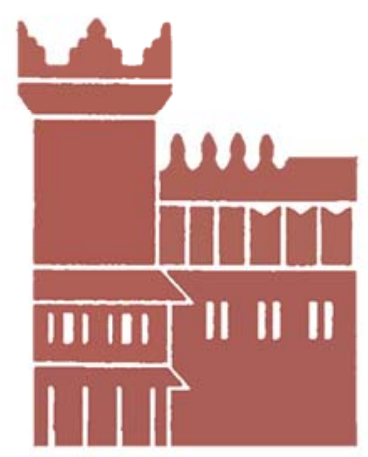

Alma Mater Studiorum - Università di Bologna DEPARTMENT OF ECONOMICS

Strada Maggiore 45

40125 Bologna - Italy

Tel. +39051 2092604

Fax +390512092664

http://www.dse.unibo.it 